\title{
Orientação estratégica para o mercado: um estudo de caso no sistema crédito cooperativo sicredi do rio grande do sul
}

\author{
Verner Luis Antoni (UPF) antoni@upf.br \\ Cláudio Damacena (UNISINOS) damacena@uol.com.br \\ Roberto Luis Frumi (UPF) frutoal@megasysnet.com.br
}

\begin{abstract}
Resumo
Estudos desenvolvidos em diferentes culturas e organizações apontam uma forte relação entre orientação para o mercado e desempenho organizacional. Partindo desse pressuposto, o artigo descreve como ocorre a orientação para o mercado numa organização de serviços que tem com premissa básica uma visão sistêmica de organização sustentada por uma filosofia associativa, o Sistema de Crédito Cooperativo Sicredi. A partir de uma abordagem qualitativa e quantitativa, foram verificadas as percepções nos diferentes níveis funcionais e as barreiras para orientação ao mercado na organização. A análise dos dados permitiu identificar que o sistema está focado em responder ao mercado, gerando e disseminando pouca inteligência de mercado. As análises bivariada e multivariada (regressão logística) dos dados permitiram identificar que nos diversos níveis hierárquicos ocorrem percepções diferenciadas sobre o grau de orientação para o mercado e as barreiras para sua efetivação, bem como foram identificadas as variáveis que mais contribuem para uma alta ou baixa orientação para o mercado nos diversos níveis hierárquicos. Dentre as barreiras identificadas observa-se um "esquecimento do mercado" e uma "baixa disseminação da inteligência do mercado".
\end{abstract}

Palavras-chave: Orientação para mercado; Sicredi; Sistema cooperativo.

\section{Introdução}

O tema orientação para o mercado, por muitos anos, tem sido considerado como uma prioridade de pesquisa pelo Marketing Science Institute (CANO, CARRILLAT e JARAMILLO, 2004), visto que tem forte influência no conceito de marketing e, por conseqüência, nas estratégias e gerenciamento mercadológico. Estudos desenvolvidos em diferentes culturas e organizações apontam uma forte relação entre orientação para o mercado e desempenho organizacional, dentre os quais podem ser citados os de Kohli e Jaworski (1990), Jaworski e Kohli (1993), Narver e Slater (1990), Day (1994, 2001), Deshpandé e Farley (1996, 1998), Appiah-Adu, (1997), Cano, Carrillat e Jaramillo, (2004). Ademais, muitos estudos investigam as barreiras para que as organizações se orientem para o mercado. Destaca-se que esses estudos contemplam não apenas empresas tradicionais de manufatura ou varejo, mas têm ampliado seu escopo para organizações de serviços hospitalares (RAJU e LONIAL, 2001), organizações que não visam o lucro (LIAO, FOREMAN E SARGENT, 2001; IGNÁCIO et al., 2002), organizações culturais (GAINER E PADANYI, 2002) e organizações de ensino superior (CARUANA, RAMASESHAN E EWING, 1996, FALEIRO, 2001 e ANTONI, 2004), as quais são cada vez mais atuantes e estão em pleno crescimento na sociedade pós-globalização.

Nesse contexto de diferentes tipologias organizacionais é desenvolvido o estudo apresentado no artigo, tendo como organização escolhida o SICREDI - Sistema de Crédito Cooperativo através de suas cooperativas de crédito singulares, atuantes no Rio Grande do Sul. Os objetivos propostos no estudo são: (1) mensurar o grau de orientação ao mercado do Sistema Sicredi; (2) identificar as percepções dos diferentes níveis hierárquicos e possíveis barreiras 
quanto à orientação para o mercado no Sistema Sicredi. O artigo está organizado da seguinte forma: a introdução, referencial teórico, descrição da metodologia utilizada e, por fim, resultados das análises e as conclusões.

\section{Orientação para o mercado e desempenho organizacional}

Naver e Slater (1990) consideram a orientação para o mercado como uma cultura empresarial que estimula comportamentos necessários à criação de valor superior ao cliente, proporcionando, com isso, vantagens competitivas às empresas em relação a seus concorrentes, através de um maior valor agregado junto aos clientes. Nessa mesma perspectiva, Kohli e Jaworski (1990) destacam que a implementação do conceito de marketing, que visa ao planejamento das ações empresariais a partir do consumidor, passa pela necessidade de se estar orientado para o mercado. Segundo Deshpandé e Farley (1998), a orientação para o mercado é uma medida que emergiu como um importante elemento para prever o desempenho numa perspectiva de sucesso no longo prazo (DESHPANDÉ; FARLEY, 1998). Em vários estudos relacionados à orientação para o mercado e desempenho organizacional pode ser observada uma perspectiva cross cultural (diferentes ambientes, culturas e organizações). Como exemplos mais relevantes podem ser citados os trabalhos de Narver e Slater (1990), Deshpandé, Farley e Webster (1993), Slater e Narver (1994), Pelham e Wilson (1996), Caruana, Ramaseshan e Ewing (1996), Pitt, Caruana e Berthon (1996), Egeren e O'Connor (1998), Appiah-Adu (1997), Steinmman, Deshpandé e Farley (2000), Raju e Lonial (2001), os quais constataram a associação positiva entre orientação para o mercado e desempenho organizacional.

No Brasil, estudos desenvolvidos por Sampaio (2000), Menna (2001), Trez (2000), Perin e Sampaio (2001), Faleiro (2001), Perin (2002) e Antoni (2004) buscaram identificar a relação entre orientação para o mercado e desempenho empresarial. A maioria desses estudos evidenciou também a relação positiva entre esses construtos.

Dentre os estudos mais recentes destaca-se o desenvolvido por Cano, Carrillat e Jaramillo (2004), o qual através do método de meta análise, verificou em cinco continentes a relação entre orientação para o mercado e desempenho. Segundo os autores, fica clara a importância da orientação para o mercado como fonte de vantagem competitiva, sendo essa uma estratégia indispensável para mercados globais e contextos cross cultural. Liao, Foreman e Sargent (2001), Ignácio et al. (2002), Gainer e Pandavi (2002) e Matsumo, Mentzer e Ozsomer (2002) ampliam o escopo de conhecimento sobre a orientação para o mercado em diferentes tipos de organizações, chegando às que não visam a lucro e de cultura empreendedora. Os autores concordam quanto à importância da orientação para o mercado também em organizações que não visam o lucro, desde que observados seus stakeholders específicos e respeitados seus objetivos e missões institucionais.

\section{Barreiras para a implementação da orientação para o mercado}

As percepções sobre barreiras que afetam tanto a implementação quanto a manutenção da orientação para o mercado de Jaworski e Kohli (1993), Slater e Narver (1995), Hurley e Hult (1998), Harris (1999), Bisp (1999), Harris e Piercy (1999), Day (2001) e Harris e Ogbonna (2001), apresentadas na Tabela 1, podem ser vistas como complementares e com muitos aspectos em comum. Ao se analisar essas barreiras verifica-se a indicação da importância de ser criar um ambiente que promova o comprometimento de todos os níveis organizacionais, através de lideranças participativas, para que, com isso, ocorram a implementação e manutenção de uma maior orientação para o mercado. 


\begin{tabular}{|c|c|c|}
\hline Autor & Enfoque Conceitual & Barreiras \\
\hline $\begin{array}{l}\text { Jaworski e } \\
\text { Kohli (1993) }\end{array}$ & $\begin{array}{l}\text { Alto gerenciamento, sistemas de } \\
\text { recompensas, centralização e } \\
\text { conexões organizacionais. }\end{array}$ & $\begin{array}{l}\text { Conflito departamental; Falta de formalização e regras } \\
\text { devidamente elaboradas de como implementar a orientação } \\
\text { para o mercado. }\end{array}$ \\
\hline $\begin{array}{l}\text { Slater e } \\
\text { Narver (1995) }\end{array}$ & Orientação para a aprendizagem. & $\begin{array}{l}\text { Incapacidade de aprendizagem da organização; Barreiras } \\
\text { funcionais que impedem o fluxo de informações } \\
\text { interdepartamentais. }\end{array}$ \\
\hline $\begin{array}{l}\text { Hurley e Hult } \\
\text { (1998) }\end{array}$ & $\begin{array}{l}\text { Relação entre inovação, } \\
\text { orientação para o mercado e } \\
\text { aprendizagem organizacional. }\end{array}$ & $\begin{array}{l}\text { Barreiras culturais diminuem a capacidade de inovação e } \\
\text { aprendizado das organizações. }\end{array}$ \\
\hline Harris (1999) & $\begin{array}{l}\text { Processo contínuo (continuum) e } \\
\text { visão holística. }\end{array}$ & Pessoas (hearts and minds) e o sistema organizacional. \\
\hline Bisp (1999) & $\begin{array}{l}\text { Forma de administração da } \\
\text { empresa. }\end{array}$ & $\begin{array}{l}\text { Personalidade da administração; Crenças individuais; } \\
\text { Fatores da estrutura organizacional; Administração dos } \\
\text { recursos humanos; Falta de competência; Clima psicológico. }\end{array}$ \\
\hline $\begin{array}{l}\text { Harris e } \\
\text { Piercy (1999) }\end{array}$ & Gerência organizacional. & $\begin{array}{l}\text { Comportamentos gerenciais formais, conflituosos ou } \\
\text { motivados politicamente; Falta de profissionalização } \\
\text { gerencial. }\end{array}$ \\
\hline Day (2001) & $\begin{array}{l}\text { Concepções errôneas do que é } \\
\text { orientar-se para o mercado. }\end{array}$ & $\begin{array}{l}\text { Esquecer o mercado; Tornar-se forçado pelo cliente; } \\
\text { Sentir-se superior ao cliente. }\end{array}$ \\
\hline $\begin{array}{l}\text { Harris e } \\
\text { Ogbonna } \\
(2001)\end{array}$ & Estilo de liderança (ownership). & $\begin{array}{l}\text { Principais barreiras são as comportamentais (intangíveis); } \\
\text { Foco demasiado em identificar barreiras tangíveis (sistema, } \\
\text { estratégia, estrutura e procedimentos organizacionais); } \\
\text { Lideranças instrumentais. }\end{array}$ \\
\hline
\end{tabular}

Fonte: Antoni, 2004.

Tabela 1 - Barreiras na implementação da orientação para o mercado.

\section{O Sistema de Crédito Cooperativo (Sicredi) e a Orientação para Mercado}

No sistema financeiro nacional, de acordo com dados do Banco Central (BACEN, 2005), em dezembro de 2003, as cooperativas de crédito participavam com 1,82\% do total de depósitos bancários do país, incluindo-se nesse montante o Sicredi. Já no Rio Grande do Sul, conforme dados do Bacen (2005), com base em outubro de 2004, a participação do Sicredi no sistema financeiro, em depósitos totais, soma $6,82 \%$. Quando se exclui a região metropolitana de Porto Alegre, essa participação sobe para mais de 15\%, demonstrando a importância econômica como agente de crédito cooperativo junto aos seus associados e sociedade. $\mathrm{O}$ desenvolvimento de uma aptidão de relacionamento com o mercado mais elevada que a dos rivais depende da disposição para mudar suas mentalidades e seus comportamentos para adquirir conhecimento total a respeito de seus clientes mais valiosos e integrar e alinhar seus processos mais importantes (DAY, 2001). Encontra-se aqui um dos grandes diferenciais que o setor de crédito cooperativo pode aliar quando da obtenção de um maior grau de orientação ao mercado, pois, além do relacionamento preconizado por essa prática, as cooperativas têm na sua essência a filosofia associativa que, se administrada corretamente, pode gerar um grau de sinergia tamanho na relação organização e mercado, que a sua condição perante a concorrência se torna praticamente insuperável.

Numa perspectiva setorial, observa-se uma literatura escassa sobre estratégia de marketing para o setor cooperativo, ainda mais se tratando de cooperativas de crédito, condição essa que motiva esse tipo de estudo pelas particularidades de origem e cultura (filosofia associativa), e estrutura (altamente hierarquizada) desse tipo de organização, bem como pela velocidade e severidade que a competição vem atingindo o setor.

\section{Procedimentos metodológicos}

Tendo como base o referencial teórico, inicialmente foram definidas as variáveis que deveriam ser mensuradas. Assim, utilizou-se a escala Markor, desenvolvida por Kohli, 
Jaworski e Kumar (1993), que definem orientação para mercado como a geração de inteligência de mercado, disseminação dessa inteligência e capacidade de resposta da empresa em relação ao mercado. Dessa forma, vinte variáveis foram medidas através dessa escala, sendo as primeiras seis relativas à dimensão "geração de inteligência de mercado", as cinco seguintes $(07,08,09,10$ e 11), relativas à dimensão "disseminação da inteligência de mercado" e a terceira dimensão "resposta ao mercado", foi medida pelas últimas nove variáveis: $12,13,14,15,16,17,18,19$ e 20 . No estudo foi utilizada uma escala Likert de sete pontos associada as variáveis, em relação às quais o respondente deveria manifestar-se de acordo com o comportamento da organização entre (1) discordo totalmente a (7) concordo totalmente. Conforme Caruana, Ramaseshan e Ewing (1996) e Antoni (2004), neste estudo optou-se pela utilização de sete pontos na escala com o objetivo de obter mais precisão quanto à intensidade com que a pessoa concorda ou discorda da afirmação (HAIR et al., 2005).

A população de pesquisa foi constituída de 632 gestores do Sistema Sicredi do Rio Grande Sul, divididos em grupos de acordo com o cargo/função que ocupam na organização, conforme exposto na Tabela 2. O questionários foram enviados a esses gestores via malote interno acompanhados de uma carta esclarecendo os objetivos da pesquisa e com a aprovação dos dirigentes do sistema para sua realização. O período de coleta ocorreu entre 17 a 30 de dezembro de 2004. Retornaram 146 questionários (23,10\% da população), das quais três apresentaram erro de preenchimento e foram invalidados, restando 143 para a amostra.

\begin{tabular}{|c|c|c|c|c|}
\hline Cargo/função & População & $\%$ & Amostra & $\%$ \\
\hline Presidente e diretores da confederação & 4 & 0,63 & 3 & 2,10 \\
\hline Gerentes da confederação & 14 & 2,22 & 9 & 6,29 \\
\hline Gerentes da corretora & 3 & 0,47 & 3 & 2,10 \\
\hline Presidente e diretores do Bansicredi & 5 & 0,79 & 5 & 3,50 \\
\hline Gerentes do Bansicredi & 10 & 1,58 & 9 & 6,29 \\
\hline Presidente, diretores, superintendentes e gerentes da Central RS & 7 & 1,11 & 6 & 4,20 \\
\hline Gerentes regionais das cooperativas & 53 & 8,39 & 7 & 4,90 \\
\hline Presidentes das cooperativas & 53 & 8,39 & 6 & 4,20 \\
\hline Gerentes de controladoria das cooperativas & 53 & 8,39 & 7 & 4,90 \\
\hline Gerentes das unidades de atendimento das cooperativas & 430 & 68,04 & 88 & 61,54 \\
\hline Total & 632 & 100,00 & 143 & $\mathbf{1 0 0 , 0 0}$ \\
\hline
\end{tabular}

Fonte: Elaborado pelos autores a partir das informações do Sistema Sicredi (2004)

Tabela 2 - População e amostra estudada

Para analisar a representatividade da amostra, foram utilizadas duas técnicas para estimar o viés de não respondentes (ARMSTRONG; OVERTON, 1977): o método de extrapolação e a técnica de comparação de dados conhecidos da população. No método de extrapolação, caracterizado pela comparação dos dados dos primeiros respondentes com os dados dos últimos, os resultados não apresentaram diferenças significativas em nenhuma das vinte variáveis que formam o construto Orientação para o Mercado. Quanto à técnica de comparação dos dados da amostra com a população, o resultado do teste não paramétrico quiquadrado mostrou-se significativo, ou seja, que há diferenças na composição da variável cargo ou função ao comparar-se a população e amostra. Como o resultado desse teste foi significativo, decidiu-se por ponderar (post-estratification) os resultados com o objetivo de corrigir esse desequilíbrio amostral (MALHOTRA, 2001; SUDMAN, 1994, TRYFOS, 1996).

Para verificar a confiabilidade da escala foi utilizado o coeficiente alfa de Cronbach, segundo vários autores (CHURCHILL, 1979, HAIR et al., 2005; MALHOTRA, 2001), o mais indicado para esse tipo de análise. Os alfas de todas as dimensões apresentaram valores superiores a 7,0. "Os pesquisadores geralmente consideram um alfa 0,7 como mínimo, embora mais baixos podem ser aceitáveis, dependendo do tipo de pesquisa." (HAIR et al., 
2005, p. 200)

\section{Resultados da análise dos dados}

Conforme Tabela 3, as dimensões "geração de inteligência" e "disseminação da inteligência" apresentam médias gerais (3,9 e 4,0, respectivamente) abaixo do ponto médio da escala e da própria média geral do construto maior $(4,2)$. De maneira geral, no Sistema Sicredi ocorre uma maior preocupação em responder ao mercado, visto que a dimensão resposta para o mercado apresenta uma média de 4,6, acima da média geral e das demais dimensões do construto maior. O maior grau de orientação (acima da média geral) é atribuído pelos respondentes das entidades que dão suporte às cooperativas, diferindo apenas os gerentes regionais, ao passo que as médias abaixo da média geral são atribuídas pelos respondentes que atuam nas cooperativas.

\begin{tabular}{|c|c|c|c|c|c|c|c|c|c|c|c|c|c|}
\hline \multirow[b]{2}{*}{ Cargo ou Função } & \multirow[b]{2}{*}{$\mathbf{N}^{0}$} & \multicolumn{3}{|c|}{$\begin{array}{c}\text { Geração de } \\
\text { Inteligência de } \\
\text { Mercado }\end{array}$} & \multicolumn{3}{|c|}{\begin{tabular}{|c} 
Disseminação da \\
Inteligência de \\
Mercado
\end{tabular}} & \multicolumn{3}{|c|}{$\begin{array}{c}\text { Resposta ao } \\
\text { Mercado }\end{array}$} & \multicolumn{3}{|c|}{$\begin{array}{c}\text { Orientação par } \\
\text { mercado }\end{array}$} \\
\hline & & Média & Med. & Dp & Média & Med. & Dp & Média & Med. & Dp & Média & Med. & Dp \\
\hline Presidente e diretores da Confederação & 3 & 6,1 & - & - & 4,3 & - & - & 5,1 & - & - & 5,2 & $1-$ & - \\
\hline Gerentes da Confederação & 9 & 5,2 & 5,5 & 1,5 & 4,1 & 4,5 & 1,5 & 4,8 & 4,9 & 0,9 & 4,8 & 5,1 & 1,1 \\
\hline Gerentes da corretora & 3 & 5,4 & - & - & 4,9 & - & - & 5,9 & - & - & 5,5 & - & - \\
\hline Presidente e diretores do Bansicredi & 5 & 4,4 & 5,9 & 4,4 & 5,0 & 5,8 & 3,6 & 5,3 & 6,4 & 3,3 & 5,0 & 5,8 & 3,4 \\
\hline Gerentes do Bansicredi & 9 & 4,7 & 5,7 & 1,7 & 4,5 & 5,1 & 1,3 & 5,8 & 6,0 & 0,8 & 5,2 & 5,5 & 1,0 \\
\hline Presidente, diretores, superintendentes e gerentes & 6 & 4,5 & 5,1 & 1,8 & 3,4 & 3,8 & 1,7 & 4,3 & 5,2 & 1,4 & 4,1 & 4,8 & 1,3 \\
\hline Gerentes regionais das cooperativas & 7 & 3,9 & 3,8 & 1,0 & 4,7 & 4,8 & 1,1 & 4,7 & 4,7 & 1,1 & 4,4 & 4,8 & 0,9 \\
\hline Presidentes das cooperativas & 6 & 3,4 & 3,6 & 1,2 & 3,7 & 3,8 & 1,7 & 4,5 & 4,6 & 1,4 & 4,0 & 4,2 & 1,4 \\
\hline Gerentes de controladoria das cooperativas & 7 & 3,5 & 3,7 & 0,9 & 3,8 & 3,8 & 1,5 & 4,4 & 4,4 & 1,0 & 4,0 & 4,2 & 1,1 \\
\hline Gerentes das agências das cooperativas & 88 & 3,9 & 4,0 & 1,2 & 4,0 & 4,0 & 1,3 & 4,5 & 4,6 & 1,1 & 4,2 & 4,3 & 1,0 \\
\hline Total & 143 & 3,9 & 4,0 & 1,2 & 4,0 & 4,2 & 1,3 & 4,6 & 4,6 & 1,1 & 4,2 & 4,4 & 1,1 \\
\hline
\end{tabular}

Fonte: Análise dos dados, 2004

* No cálculo da média de OM foram consideradas todas as questões, e não apenas as médias das dimensões.

Tabela 3 - Grau médio, mediano e desvio padrão das dimensões de Orientação ao Mercado em função do cargo do respondente.

Convém salientar que as cooperativas estão em contato direto com os associados e clientes na prestação de serviços; as demais desenvolvem estratégias de negócios e produtos, bem como dão suporte operacional, observando-se atribuições de cada uma. Esse ponto reforça a dificuldade em se disseminar as informações dentro das diferentes estruturas do sistema, bem como uma disfunção organizacional na perspectiva de orientação para o mercado, visto que os níveis hierárquicos mais distantes dos mercados são responsáveis por estratégias e produtos, sem ter informações efetivas e mesmo um fluxo consistente de informações sobre a percepção do ambiente mercadológico, dando-se destaque a capacidade de responder às mudanças do mercado e ouvir aos associados e clientes.

\subsection{Análise multivariada dos dados}

A técnica estatística multivariada regressão logística foi utilizada para identificar as variáveis mais importantes para explicar as diferenças nos níveis de orientação para mercado. Para tanto, foi aplicado o método passo-a-passo progressivo (forward stepwise), no qual é utilizada a diferença do qui-quadrado para determinar automaticamente quais variáveis são adicionadas ou retiradas do modelo (GARSON, 2005). Desta forma, as variáveis selecinadas foram: (Var4) nós pesquisamos os associados e clientes pelo menos uma vez ao ano para avaliar a qualidade de nossos serviços; (Var8) o pessoal de marketing em nossa organização investe tempo discutindo com outros departamentos sobre as necessidades futuras dos associados e clientes; (Var11) quando um departamento descobre algo importante sobre os concorrentes, 
ele é rápido para alertar todos os outros departamentos; (Var12) somos rápidos para decidir como responder às mudanças de preços (taxas de aplicações, crédito, tarifas, seguros, ...) dos nossos concorrentes; (Var14) nós revisamos periodicamente nossos esforços de desenvolvimento de novos produtos e serviços para assegurar que eles estão de acordo com o que os associados e clientes desejam; (Var15) diversos departamentos encontram-se periodicamente para planejar uma resposta às mudanças que ocorrem em nosso ambiente de negócio; (Var20) quando sabemos que os associados e clientes gostariam que modificássemos um produto ou serviço, os departamentos envolvidos fazem esforços combinados para afetar a modificação. Essas variáveis indicaram uma precisão de $95,1 \%$ no modelo de regressão logística, o que explicita a qualidade do resultado dessa análise.

As diferenças de percepção somadas a uma capacidade reduzida de disseminação da inteligência, podem promover barreiras para a implementação ou, ao menos, para o desempenho efetivo em termos de desempenho organizacional (JAWORSKI e KOHLI , 1993), visto que ao se observar as análises ocorre um indicação da falta de formalização ou de regras claras de como implementar a orientação para o mercado. A análise indica que uma das principais barreiras esteja relacionado ao que Day (2001) chama de "esquecer o mercado", ou seja, que a organização esteja centrada no produto ou serviço que oferece, ficando voltada para dentro, o que leva a não ter uma visão clara do mercado. Segundo Day (2001), quando isso ocorre, a organização pode ter sido orientada para o mercado, mas só percebe que perdeu seu foco quando enfrenta problemas.

\section{Conclusões e implicações}

Os resultados do estudo apontam que o Sistema de Crédito Cooperativo - Sicredi apresenta um grau de orientação para o mercado um pouco acima do ponto intermediário da escala, atingindo 4,2. De maneira geral, a organização está mais voltada a responder para o mercado do que a gerar e disseminar inteligência de mercado.

Numa perspectiva teórica de ampliar o corpo de conhecimento sobre orientação para o mercado e barreiras para sua implementação, deve ser destacado que, ao se estudar o conjunto de cooperativas, que apresenta particularidades culturais, estruturais e funcionais diferentes de outros modelos de organização, comprovou-se que a escala Markor foi adequada ao contexto estudado, bem como a literatura sobre tema deu suporte para as análises na organização pesquisada. Em termos de ampliação do escopo na área de conhecimento, o artigo contribuiu no estudo de uma organização no setor de serviços e que não visa a lucro, uma "cooperativa de crédito", que tem no seu associado o foco de mercado, bem como apresenta como premissa básica uma visão sistêmica de organização, sustentada pelo relacionamento junto ao associado, ou seja, sua essência é baseada na filosofia da associativa.

Gerencialmente, constatou-se que ocorrem percepções diferentes entre os diversos níveis hierárquicos da organização, ao mesmo tempo em que prevalece um comportamento nos diversos níveis a responder para o mercado, indicando uma disfunção funcional na perspectiva de orientação para o mercado, pois os níveis superiores que traçam as estratégias e desenvolvem produtos e serviços consideram escores relativamente altos de orientação para o mercado, por sua vez os níveis gerencias e operacionais mais próximos do mercado sentem um distanciamento dos associados e clientes. Isso, somado a um índice relativamente baixo de geração e disseminação da inteligência, conduz a uma postura reativa para com o mercado e o desenvolvimento de produtos e serviços numa perspectiva internalizada, o que a médio e longo-prazo, pode gerar conflitos culturais na organização e perda de competitividade do sistema como um todo. Quanto às limitações do estudo, relacionam-se a se tratar de um caso, não permitindo generalizações para outras organizações do mesmo tipo, bem como seu escopo se limitou a mensurar e identificar barreiras de orientação para o mercado numa 
organização de serviços, cooperativa, e sem fins lucrativos. Essa opção não considerou a verificação de medidas de desempenho e sua relação com orientação para o mercado. Estudos futuros poderão, numa perspectiva cross organizacional, aplicar essa metodologia em outras cooperativas para propor um construto específico para esse tipo de organização tão presente no contexto brasileir. Outra sugestão de pesquisa está relacionada com a análise da existência de um gap entre o fornecedor de serviços (Cooperativa) e seus associados e clientes, como sugerem Steinman, Deshpandé e Farley (2000), considerando, sobretudo, o tipo de organização, no qual a filosofia associativa é imprescindível.

\section{Referências}

ANTONI, V. L. (2004) - A relação entre orientação para o mercado e desempenho organizacional: um estudo nos cursos de bacharelado em administração da região Sul do Brasil. Tese (Doutorado), UFSC, Florianópolis.

APIAH-ADU, Kwaku (1997)- Market orientation and performance: do the findings established in large firms hold in the small business sector? Journal of Euro-Marketing, v. 6.

ARMSTRONG, S. J.; OVERTON, T.S. (1997) - Estimating non-responses bias in mailed surveys. Journal of Marketing Research, Vol. 13, p. 396 - 402, Aug.

BACEN - BANCO CENTRAL DO BRASIL. (2005) - Página Institucional. Disponível em: $<$ http://www.bcb.gov.br>. Acesso em: jan.

BISP, Soren. (1999) - Barriers to increased market-oriented activity: what the lierature suggests. Journal of Market-Focused Management, v. 4.

CANO, R. C; CARRILLAT, F. A.; JARAMILLO, F. (2004) - A meta-analysis of the relationship between market orientation and business performance: evidence from five continents. International Journal of Research in Marketing, Vol. 21.

CARUANA, Albert.; RAMASESHAN, B.; EWING, Michael.(1996) - Market orientation and performance: a study of Australasian Universities. Curtin Business School. Working Paper Series, Nov.

CHURCHILL, G. A. (1979) - A paradigm for developing better measures of marketing constructs. Journal of Marketing Research. v. 26, p. 64-73.

DAY, G. S. The capabilities of market-driven organizations. Journal of Marketing, v. 58, p. 37 - 52, Oct. 1994.

DAY, George S. (2001) - A empresa orientada para o mercado: compreender, atrair e manter clientes valiosos. Porto Alegre: Bookman.

DESHPANDÉ, R.; FARLEY, J. (1998) - Understanding market orientation: a prospectively designed metaanalysis of the three market orientation scales. MSI, 1996. In: DESHPANDÉ, Rohit; FARLEY, John U. Measuring Market Orientation. Generalization and Synthesis. Journal of Market-Focused Management, v. 2.

DESHPANDÉ, R.; FARLEY, J. U.; WEBSTER, F. E. Jr. (1993) Corporate culture, customer orientation, and inovativeness in Japanese firms: a quadrad analysis. Journal of Marketing. V. 57, p. 23 - 27, Jan.

DESHPANDÉ, Rohit; WEBSTER, Frederick E. Jr (1989) - Organizational culture and marketing: defining the research agenda. Journal of Marketing, v. 57, p. 3 - 15, Jan.

EGEREN, Van Marsha; O'CONNOR, Stephen. (1998) - Drivers of market orientation and performance in service firms. Journal of Services Marketing, v. 12, n. 1, p. 39 - 58.

FALEIRO, Sandro N. (2001) - A relação entre orientação para o mercado, orientação para a aprendizagem e inovação. $O$ caso dos cursos de graduação em administração filiados a Angrad.. Dissertação (Mestrado em Administração) - PPGA / Escola de Administração, UFRGS, Porto Alegre.

GAINER, Brenda; PANDANYI, Paulette. (2002) - Applying the marketing concept to cultural organisations: an empirical study of the relationship between market orientation and performance. International Journal of Nonprofit and Voluntary Sector Marketing, Vol. 7, p. 182 - 193, May.

GARSON, D. (20/03/05) - PA 765 Statnotes: An Online Textbook. Disponível na internet: http://www2.chass.ncsu.edu/garson/pa765/statnote.htm .

HARRIS, L. C. (1999) - Barriers to developing market orientation. Journal of Aplied Management Studies, v. 8.

HARRIS, L. C.; OGBONNA E. (2001) - Leadership style and marketing orientation: An empirical study. 
European Journal of Marketig, v. 35.

HARRIS, Loyd C.; PIERCY, N. F. (1999) - Management behavior and the barriers to market orientation. Journal of Services Marketing, v.13.

HURLEY, Robert F.; HULT, G. Tomas M. (1998) Innovation, market orientation, and organizational learning: an integration and empirical examination. Journal of Marketing, v. 62, p. 42 - 54, July.

IGNÁCIO, L. et al. (2002) - The market orientation concept in the private nonprofit organisation domain. International Journal of Nonprofit and Voluntary Sector Marketing, v.7, p. 55 - 67, Feb.

KOHLI, Ajay K.; JAWORSKI, Bernard J. (1990) - Market orientation: the construct, research propositions, and managerial aplications. Journal of Marketing, v. 54, p. 1 - 18, Apr.

KOHLI, Ajay K; JAWORSKI, Bernard J.; KUMAR, Ajith. (1993) - Markor: a measure of market orientation. Journal of Marketing, v. XXX, p. 467 - 477, Nov.

LIAO, Mei-Na; FOREMAN, Susan; SARGEANT, Adrian. (2001) - Market versus societal orientation in the nonprofit context. International Journal of Nonprofit an Voluntary Sector Marketing. London, Sep..

MALHOTRA, N. K. (2001) - Pesquisa de marketing: uma orientação aplicada. $3^{\mathrm{a}}$ ed. Porto Alegre: Bookman.

MATSUNO, Ken; MENTZER, John T.; OZSOMER, Aysegul (2002) - The effects of entrepreneurial proclivity and market orientation on business performance. Journal of Marketing, v. 66, p. 18 - 32, July.

MENNA, Hélio L. (2001) - Orientação para mercado e performance: evidências em empresas gaúchas de varejo de confecções masculinas. In: ENANPAD, 25, 2001, Campinas (SP). Anais... Campinas: ANPAD.

NARVER, John C.; SLATER, Stanley F. (1990) - The effect of market orientation on business profitability. Journal of Marketing, v. 54, p. 20 - 35, oct.

NARVER, J. C; SLATER, S. F.; TIETJE, B. (1998) - Creating a market orientation. Journal of Market-Focused Management, v. 2.

PELHAM, Alfred M.; WILSON, David T. (1996) - A Longitudinal Study of the Impact of Market Structure, Firm Structure, strategy, and Market Orientation Culture on Dimensions of Small-Firm Performance. Journal of Academy of Marketing Science, v. 24, n.1, p. 27 - 43, Winter.

PERIN, M. G. (2002) A relação entre orientação para mercado, aprendizagem organizacional e performance. Tese (Doutorado em Administração) - PPGA / Escola de Administração, UFRGS, Porto Alegre.

PERIN, Marcelo G.; SAMPAIO, Cláudio H. (2001) - A relação entre as dimensões de orientação para o mercado e a performance. In: ENANPAD, 25, 2001, Campinas (SP). Anais...Campinas: ANPAD.

PITT, Leyland; CARUANA, Albert; BERTHON, Pierre R. (1996) - Market orientation and business performance: some European evidence. International Marketing Review, v. 13.

RAJU, P. S. e LONIAL, Subhash (2001) - The impact of quality context and market orientation on organizacional performance in a service environment. Journal of Service Research, v. 4, p. 140 - 154, Nov.

RAJU, P.S. ; LONIAL, Subhash; GRUPTA, Yash P. (1995) - Market Orientation and Perfromance in the Hospital Industry. Journal of Health Care Marketing, v. 15, p. 34 - 41, Winter.

SAMPAIO, Claúdio H. (2000) - Relação entre orientação para o mercado e performance empresarial em empresas de varejo de vestuário do Brasil. Tese (Doutorado em Administração) - PGA / Escola de Administração, UFRGS, Porto Alegre.

SLATER, S. F.; NAVER, J. C. (1995) - Market orientation and the learning organization. Journal of Marketing, v. 59 , p. $63-74$, July.

SLATER, Stanley F.; NARVER, John C. (1994) - Does competitive environment moderate the market orientation performance relationship? Journal of Marekting, v. 58, p. 46 - 55, Jan.

STEINMAN, Christie; DESHPANDÉ, Rohit; FARLEY, John U (2000) - Beyond market orientation: when customers and supliers disagree. Academy of Marketing Science, Greenvale, Winter.

SUDMAN, S. (1994) - Sampling. In: Bagozzi, R. P. Principles of Marketing Research. Cambridge: Blackwell.

TREZ, Guilherme (2000) - Relação entre orientação para serviços ao cliente e performance das organizações.. Dissertação (Mestrado em Administração) - PPGA / Escola de Administração, UFRGS, Porto Alegre.

TRYFOS, P. (1996) - Sampling methods for applied research: text and cases. Nova York: John Wiley \& Sons. 